Service social

\title{
Intervention et stratégies familiales en interculturel
}

\section{Michèle Vatz-Laaroussi}

Volume 42, numéro 1, 1993

\section{Culture et intervention}

URI : https://id.erudit.org/iderudit/706599ar

DOI : https://doi.org/10.7202/706599ar

Aller au sommaire du numéro

Éditeur(s)

École de service social de l'Université Laval

ISSN

1708-1734 (numérique)

Découvrir la revue

Citer cet article

Vatz-Laaroussi, M. (1993). Intervention et stratégies familiales en interculturel. Service social, 42(1), 49-62. https://doi.org/10.7202/706599ar

\section{Résumé de l'article}

Si les recherches sur les immigrants portent sur leurs liens avec les communautés ethniques d'origine, sur leurs stratégies individuelles d'adaptation au pays d'accueil ou encore sur leurs groupes élargis d'appartenance (hommes, femmes, jeunes, travailleurs...), la dynamique familiale du changement est le plus souvent passée sous silence ou a priori considérée comme un frein à l'intégration sociale.

Une revue des recherches effectuées en Europe et au Québec ainsi que les résultats d'une enquête exploratoire au Québec permettent de donner un premier inventaire des stratégies familiales d'acculturation et de survie dans l'immigration. L'analyse et la conscientisation de ces stratégies forment alors les bases d'un modèle d'intervention familiale interculturelle. 


\section{Intervention et stratégies familiales en interculturel}

Michèle VATZ LAAROUSSI

Professeure

Département de service social Université de Sherbrooke

\section{INTRODUCTION}

Si le travail social est avant tout la rencontre de l'autre à la fois dans sa spécificité et dans sa globalité, alors l'intervenant devrait être le champion de l'interculturel.

Et pourtant au Québec, comme dans tous les pays industrialisés où le travail social a développé de nombreux modèles et de multiples techniques, le contexte multiculturel vient interroger, remettre en question, «remuer » I'intervention tout comme l'intervenant.

Il est aisé de constater combien l'intervention, I'action sociale se situent souvent dans le malentendu culturel. Il semble bien que les différentes approches sociales se heurtent à la polysémie du message et de l'intervention en milieu multiculturel.

L'immigration met en œuvre, de manière collective et individuelle, une dynamique, une complexité, un mouvement tels que les différents acteurs vont en être déstabilisés.

L'immigrant, le réfugié, le noir, l'étranger vont avoir à se définir et se redéfinir dans un monde où l'habituel devient exceptionnel, où l'anormal devient la règle. 
Le «qui suis-je? » existentiel prend alors un sens nouveau qui peut être celui du deuil, mais aussi du changement ou de l'innovation.

Et pour aborder cette question et les réponses que chacun y trouve (les stratégies), il nous paraît essentiel de passer par le cadre de vie structurel de chacun, immigrant ou Québécois de souche, le groupe primaire souvent mis dans l'ombre par la communauté ethnique: c'est de la famille que nous voulons parler.

Les différentes études parues dernièrement sur l'immigration et l'accueil au pays vont en effet porter sur les problématiques centrées sur des individus ou sur des groupes déjà secondaires (les minorités ethniques, les femmes, les jeunes, les travailleurs...) en "gommant» le fait que la plupart de ces immigrants arrivent et vivent en famille.

Cellule de base avant, pendant et, peut-être, après l'immigration, la famille immigrante se retrouve ignorée de nos recherches et interventions.

Le type d'analyse et d'intervention proposé ici reposera au contraire sur ce groupe de vie et principalement sur les stratégies que la famille met en œuvre dans l'immigration: stratégies d'acculturation et de changement sûrement, stratégies de survie le plus souvent, stratégies d'insertion parfois.

Afin d'approfondir les raisons théoriques et concrètes qui nous font privilégier cet angle de vue, nous argumenterons d'abord notre postulat $d^{\prime}$ 'envisager la famille comme un groupe essentiel dans le processus $d^{\prime}$ 'immigration. Le deuxième temps de cette démonstration tentera de construire un questionnement autour des deux sous- systèmes, famille/communauté ethnique, permettant ensuite d'envisager concrètement une typologie encore incomplète des stratégies en jeu. Cette analyse et les hypothèses qui en découlent nous permettront enfin de poser les pierres angulaires d'un modèle $d^{\prime}$ intervention familiale en interculturel.

Ce modèle, lorsqu'il aura été complété et expérimenté, devra permettre au travailleur social d'approcher la complexité du vécu migratoire et d'accompagner les membres de la famille dans leur « reconstruction » psychosociale tant sur le plan individuel que collectif.

Il est à noter que la typologie des stratégies familiales est dressée à partir des recherches effectuées précédemment (Vatz Laaroussi, 1986, 1988 et 1990) et d'une recherche exploratoire en cours qui utilise une méthode qualitative de type récits de vie auprès de chaque membre de la famille (père, mère, enfants).

Ce sont les corrélations établies entre les récits de vie qui permettent de tracer un premier modèle de ces stratégies. Celles ci vont ensuite être étudiées de manière plus systématique et grâce à des outils plus pointus (budget temps, budget espace, topographie des réseaux, tests projectifs...).

Les modalités d'intervention sont, elles, perçues à travers notre pratique en quartier multiethnique en France et grâce à la recherche exploratoire en 
cours sur les modalités de l'intervention au Québec en fonction de la région d'appartenance.

\section{POURQUOI PARLER DE LA FAMILLE IMMIGRANTE?}

Un premier argument est de considérer que, dans les pays d'origine des immigrants de nos sociétés, la famille élargie est le noyau social de base qui permet à la personne d'être reconnue et intégrée à la société. Qu'il s'agisse de contextes socioculturels aussi différents que ceux trouvés en Amérique du Sud, au Moyen-Orient, en Asie, aux Antilles ou en Afrique, la famille, avec ses structures spécifiques, semble être une constante dans le mode de vie et d'organisation sociale des différentes contrées considérées (Camilleri, 1980). Les grandes religions elles-mêmes, I'islam, le christianisme, le judaïsme ou le bouddhisme, ont donné tant de poids à la structure familiale que nos cultures, mentalités et sociétés ne peuvent qu'en être imprégnées. Il en est de même pour les différents codes juridiques qui, dans chaque pays, instaurent et réglementent les droits et devoirs de chacun en fonction de son appartenance et de son statut familial.

En dehors même de cette structuration sociale établie autour de l'entité familiale, de nombreuses études tant ethnographiques que sociologiques ou psychologiques montrent que c'est dans son noyau familial que le jeune acquiert l'estime de soi, la sécurité affective et psychologique nécessaires à sa socialisation et à son développement. Ainsi, même les dernières recherches sur l'éclatement familial au Québec et aux États-Unis montrent qu'il ne s'agit pas d'une fin de la famille, mais de l'apparition de nouvelles structures familiales qui remplissent les mêmes fonctions (Fortin, 1987). Par contre, notre méconnaissance de ces nouvelles structures place nos sociétés dans une période de mutation où les coûts sociaux de l'éclatement familial sont grands (Bouchard, 1991), qu'on fasse référence à la délinquance, au décrochage scolaire, au taux de suicide des jeunes ou aux abus divers dont ils peuvent être victimes.

Les familles immigrantes au Québec vont être placées dans cette même mouvance socioculturelle et auront à payer les mêmes prix que les Québecois de souche aux changements de la famille. Mais va s'y ajouter un coût supplémentaire: celui de l'acculturation. Définie par Abou (1981), I'acculturation recouvre tous les phénomènes qui se produisent lorsque deux cultures sont en contact. Pour les immigrants, l'acculturation se fera d'abord choc culturel puis perte, rupture avant d'être la base de reconstruction identitaire Jacob, 1991). Tout se passe comme si les immigrants avaient à traverser une période d'anomie pour en sortir autres et donc innovants (Duvignaud, 1979). Mais dans cette anomie-là, le prix du changement est élevé: les familles le paieront par les suicides des jeunes filles, les fugues des garçons, les retours contraints au pays pour un mariage obligé, enfin par un taux de placement en institution 
des jeunes plus élevé chez les immigrants que chez les populations de souche (Costa Lascoux, 1986; Jasmin, 1992). II s'agit donc là d'un coût à la fois individuel (souffrance), familial (ruptures) et social (finances publiques).

Alors si le fait de s'attaquer aux problèmes des jeunes au Québec signifie, dans une grande orientation de prévention, s'attaquer aux problèmes de la famille et à ses mutations conjoncturelles ou structurelles (Bouchard, 1991), la prévention et l'intervention auprès des jeunes immigrants se devra d'autant plus de prendre en considération ce déterminant.

L'approche démographique vient nous donner une confirmation supplémentaire quant à ce «parti pris » de la famille. Les dernières statistiques (Statistique Canada, 1991) font en effet ressortir que les immigrants arrivent au Québec en famille et font venir quand ils le peuvent leur réseau familial élargi. Ce processus est vérifié tant pour les immigrants économiques que pour les réfugiés (Jacob, 1991). Ne pas tenir compte de ce réseau primaire dans nos analyses et programmes d'intervention se révèle alors être une erreur grossière par rapport à la réalité telle qu'elle est actuellement structurée. Cet argument est d'autant plus fort pour les régions périphériques où les immigrants, moins nombreux que dans la région montréalaise, semblent venir et rester seulement s'ils disposent d'un soutien familial (Le partenaire, 1993, Régie régionale de la santé et des services sociaux de l'Estrie).

Camilleri (1992), dans un survol de la situation des familles immigrées en Europe, affirme que la famille perd chez les immigrants sa qualité de groupe d'appartenance exclusive, mais qu'elle reste un pôle de référence beaucoup plus fort et plus large que chez les Européens. C'est comme si, dans le choc culturel, les immigrants rebâtissaient une entité familiale de référence qui n'est plus celle de leur pays d'origine, mais qui reste un repère dans une trajectoire de ruptures. Ainsi, dans cette même étude, Camilleri (1992) insiste sur la volonté affirmée des jeunes des secondes générations d'immigrants de sauvegarder la famille malgré tous les conflits et dysfonctions. Notre propre recherche (Vatz-Laaroussi, 1986) auprès d'immigrants en France et de Maghrébins dans leur pays d'origine montre en effet combien, dans ces histoires de vie différentes, les jeunes vont se servir de la famille comme une référence affective et sociale et ce, quelles que soient leurs dissensions et dissemblances avec les aînés. Mais cette recherche ainsi que celle effectuée auprès des chômeurs de longue durée en France (VatzLaaroussi, 1988) nous permettent de postuler que, devant le changement, les familles réagissent différemment en fonction de leur expérience, de leur trajectoire et de leur contexte de vie. II n'y a pas un type de famille immigrante: il y a une multitude de stratégies familiales face aux changements, contraintes et ruptures vécus dans l'immigration. II n'y a pas une facon de distribuer les rôles et fonctions dans le groupe familial, il y a une multitude de négociations, compromis, organisations qui gèrent la vie de chacun au quotidien. 
Et cette multitude de «façons d'être familiales » va, dans l'immigration, être porteuse des différences structurelles vécues tant au pays d'origine qu'au pays d'accueil : différences de classes sociales, différences idéologiques et politiques, différences sexuelles, différences générationnelles.

Si la notion de communauté ethnique peut amener au ghetto (Jacob et Bertot, 1991), elle peut aussi "gommer » ces différences structurelles au profit d'une vision uniforme, généralisée et donc réductrice ou même folklorique des individus d'une même ethnie. Réintroduire le réseau primaire familial dans l'analyse des situations des immigrants et pour la programmation d'interventions à leur égard, s'avère alors être une démarche écologique d'approche de l'immigrant dans ses divers environnements.

\section{FAMILLES IMMIGRANTES ET COMMUNAUTÉS ETHNIQUES AU COEUR DE LA RECONSTRUCTION IDENTITAIRE}

L'approche relativiste et comparative des systèmes juridico-légaux français et québécois (voir notre recherche en cours) montre une différence significative sur la façon dont la communauté ethnique est intégrée à la politique de l'immigration.

En France, elle n'est juridiquement pas reconnue. La communauté est vue comme une entité culturelle quasi folklorique qui ne gêne pas aussi longtemps qu'elle n'est qu'outil d'expression artistique d'une culture considérée comme traditionnelle et donc " dépassée », aisément dominée. Par contre, elle devient dangereuse lorsqu'elle déborde du cadre minoritaire et culturellement restrictif qui lui est socialement alloué. C'est là que la fameuse notion du "seuil de tolérance» intervient: quand l'association des Maghrébins commence à se faire entendre dans les médias, par le nombre de ses participants ou pour des revendications socio-économiques et politiques, la communauté ethnique, gagnant en visibilité, va se voir imposer des limites nouvelles et marquées. La revendication, le positionnement politique ne sont pas les missions légitimement tolérables de ces groupes dont le principal mandat devient alors: la restriction de la culture d'origine et des aspects structurels de l'immigration à une identité collective folklorique tout en favorisant par ailleurs l'assimilation de la différence à la culture dominante.

Au Québec, le discours politique se veut multiculturel : la différence est prônée, dite enrichissante et la communauté culturelle est présentée comme I'outil intégrateur par excellence. Cette intégration théorique correspondrait à l'enrichissement réciproque des différentes cultures en jeu par leur mise en contact. Mais c'est aussi oublier l'aspect structurel de ces cultures et de leur mise en contact: I'immigrant n'arrive pas vierge d'expérience, porteur d'une 
culture d'origine «pure ». Il vient avec son passé, ses expériences, sa décision d'immigration et ce qui l'a conditionnée. II n'arrive pas non plus dans une culture «pure»: il arrive dans une société qui a instauré une politique de I'immigration et des structures pour les immigrants en fonction de ses intérêts et conceptions (Jacob et Bertot, 1991). La communauté peut alors devenir le lieu du repli défensif de l'immigrant ballotté dans des contraintes incompréhensibles et inconciliables. Elle peut aussi être l'outil social d'une assimilation subversive, parce que non dite et même contraire au discours officiel. L'emploi par les organismes d'intervenants sociaux issus des communautés culturelles prend alors plusieurs sens.

S'agit il de travailler dans la proximité culturelle avec l'immigrant pour faire passer de manière efficace le message intégrateur?

S'agit il de se servir de cette même proximité pour lui faire sauter le pas de l'assimilation déculturante?

S'agit il enfin de le repousser et de le laisser dans la communauté ethnique sans lien avec la société dominante?

Si la réponse à ces questions dépend en grande partie des conceptions et logiques qui président à la manière dont nos institutions vont déployer et utiliser l'outil « communauté ethnique », elle est aussi très liée à la façon dont les immigrants vont le comprendre, le saisir, se l'approprier.

Camilleri (1991) définit l'identité psychosociale comme l'interface entre I'individu et ses environnements sociaux, interface qui a pour fonction de maintenir une forme de continuité dans le temps, l'unicité de son contenu et la valorisation de soi.

Malewska Peyre (1984) montre comment cette identité est, chez les jeunes, marquée par des alternances parfois contradictoires entre le «eux» et le «nous ». De même Zavalloni (1989), dans son approche égo-écologique, axe une partie de sa méthodologie sur la différenciation, la représentation et l'appropriation des groupes de pairs, d'affiliation, de référence indiquant combien cette méthode paraît riche dans le contexte de l'immigration.

La manière dont l'immigrant va subjectiver la réalité de la communauté ethnique au pays d'accueil représentera donc une composante importante de la construction-reconstruction de son identité psychosociale et ce, quels que soient son sexe ou sa génération.

Notre recherche en France (Vatz-Laaroussi, 1986) a en effet montré que si les jeunes immigrants sont en pleine construction identitaire et donc fortement soumis à l'influence des différents groupes dans la société d'accueil, la génération des parents se trouve, elle, en reconstruction identitaire et déterminée par ces mêmes forces conjuguées à celles de leurs expériences et trajectoires.

$\mathrm{Si}$, comme nous l'avons argumenté en première partie, la famille est un groupe primaire avec lequel il faut compter pour comprendre les stratégies 
mises en œuvre dans l'immigration, la communauté ethnique va sans aucun doute représenter un autre groupe de référence, qu'il s'agisse d'une référence attractive ou négative.

Mais s'il est clair que la communauté ethnique dans le pays d'accueil n'est pas une émanation «pure» du pays d'origine, ce pays lui-même, idéalisé ou revendiqué, fui ou abandonné, dévalorisé ou survalorisé, redevient un groupe «subjectivé » d'appartenance ou de référence. En ce sens les rapports réels et idéels au pays d'origine vont aussi avoir un impact sur l'interface psychosociale de l'identité des immigrants. Le test du «Qui suis je? » passé auprès de populations d'immigrants portugais et maghrébins en France (Zaleska, 1982) montre combien ces différentes références, le pays d'origine, la communauté ethnique, la famille, les institutions et groupes du pays $\mathrm{d}^{\prime}$ accueil, se trouvent intriquées dans les repères psychosociaux des jeunes.

Notre recherche de 1986 (Vatz-Laaroussi) et celle de Camilleri (1990) insistent de plus sur le fait que ces repères vont faire l'objet de négociations au sein de la famille tant à travers les modalités de l'éducation mise en œuvre par les parents que par la distribution des rôles vis à vis de l'extérieur et par enfin les différents types de modération de conflit construits par les deux générations.

Nous pouvons visualiser l'intrication de ces différents niveaux d'influence sur le schéma qui apparaît ci-après.

\section{Émergence des stratégies familiales d'acculturation}

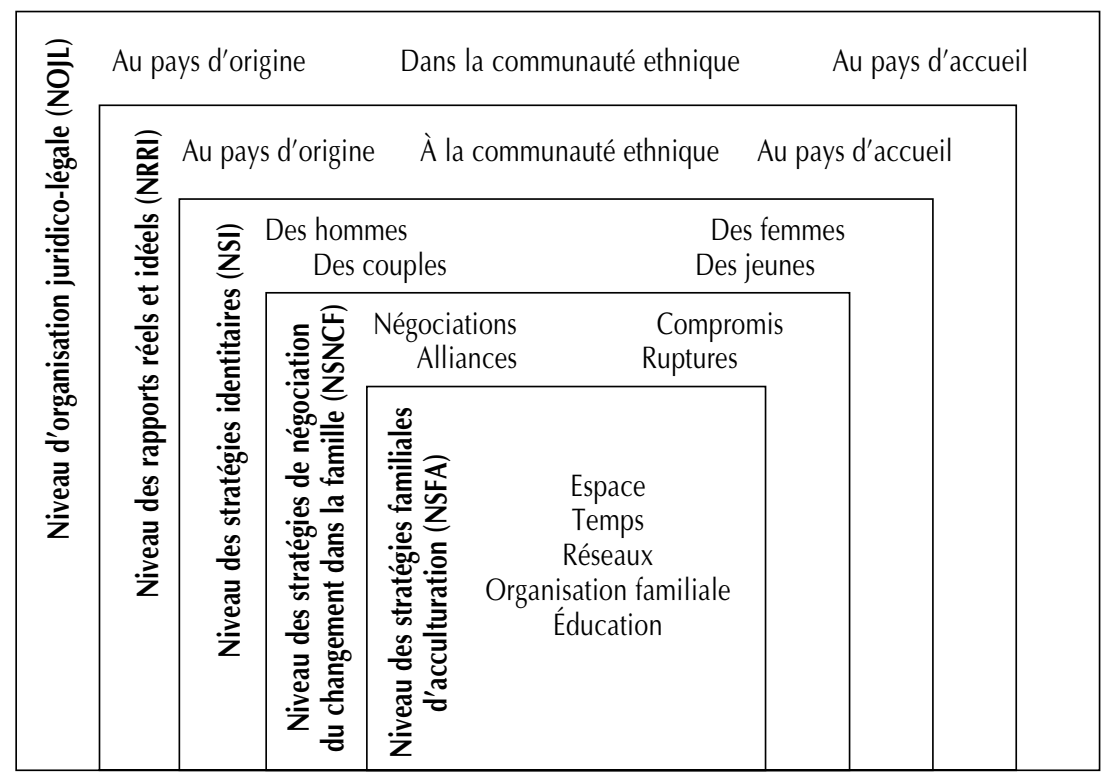




\section{LES STRATÉGIES FAMILIALES}

Nos recherches actuelles articulant ces différents niveaux prennent pour objet les stratégies familiales d'acculturation posant pour hypothèse qu'elles peuvent être un outil important d'analyse et de pratique pour l'intervenant social et pour la mise en œuvre de programmes sociaux en faveur des immigrants.

Nous entendons alors par stratégies: «le résultat de l'élaboration individuelle et collective des acteurs, exprimant dans leur mouvance les ajustements opérés au jour le jour en fonction de la variation des situations et des enjeux qu'elles suscitent, c'est-à-dire des finalités exprimées par ces acteurs et des ressources et contraintes de ceux-ci » (Camilleri, 1991).

Le niveau des stratégies identitaires a déjà été travaillé en Europe et au Québec principalement pour les adolescents, puisqu'il est démontré que c'est à cette période qu'il y a passage de l'univers relativement peu différencié de la famille à un univers social plus complexe abordé dans une perspective de plus en plus individualisée (Claes, 1991). Camilleri (1992) dresse ainsi une typologie riche de ces stratégies chez les jeunes immigrants de la seconde génération de Portugais et de Maghrébins: oscillant entre le prescrit collectif et I'autonomie de I'expérience individuelle, chacun va mettre en place des modalités de gestion de la contradiction (alternance des codes ou subjectivation-individualisation des modèles prescrits traditionnellement ou encore symbolisation identitaire d'une prescription rituelle...). De même, A. Laperrière (1992) insiste au Québec sur la différenciation des stratégies de réduction des tensions internes ou externes entre trois groupes de jeunes: des Québécois français, des jeunes d'origine italienne et des jeunes Haïtiens.

La visibilité ethnique, l'ancienneté de l'immigration et la reconnaissance de la communauté sont pointées comme des variables essentielles dans la construction de ces stratégies axées entre autres sur le pluralisme culturel, la revendication ethnique ou l'évitement du conflit. Dans cette dernière étude, tout comme dans celles de Malewska Peyre (1988) ou de Camilleri (1991), les attitudes des parents sont vues selon l'échelle Tradition / Modernisme et principalement analysées à travers le rapport des parents à la culture d'origine. Notre recherche (Vatz-Laaroussi, 1986) montrait que les stratégies d'acculturation des parents pouvaient être aussi variées que celles des jeunes et qu'elles étaient soumises aux variables de niveau socioculturel et socioprofessionnel, mais aussi aux modalités du changement culturel vécu quotidiennement (changement intrinsèque: intériorisé comme normal en lien avec le temps et les générations; changement extrinsèque: subi comme une contrainte ou une violence). S'il est clair que ces modalités du changement culturel des parents ont une influence directe sur leur système d'éducation, elles vont aussi avoir un impact sur leur manière d'organiser leur vie quotidienne d'homme, de femme, de parent, de famille, de membre de communauté. En ce sens, il va y avoir restructuration du rapport au temps, à l'espace, aux réseaux 
primaires et secondaires pour chaque individu, mais aussi pour chaque entité à laquelle il appartient (couple, famille).

Ce sont ces différents niveaux stratégiques de changement culturel qui vont s'articuler et interagir dans les négociations qui s'instaurent au sein de la famille.

Sur ce plan, les recherches de Malewska Peyre (1985), Camilleri (1992) et Vatz-Laaroussi (1986, 1988, recherche en cours) permettent d'établir une première typologie de ces modalités de négociation familiale.

On peut ainsi distinguer les stratégies de compromis (forme de marchandage dans laquelle chacun perd une partie de ses principes), les stratégies d'alliance parfois proches de la complicité (intersexe, intergénération, affectives...), les stratégies de négociation-dialogue (tenant compte des modes de communication formelle au pays d'origine et s'articulant souvent autour des notions de fierté, d'honneur et de respect) et les stratégies de rupture (dans le temps, dans l'espace, dans le réseau; ces ruptures pouvant conduire à une forme de morcellement de la famille et de ses membres).

Nous en arrivons finalement à notre objet de recherche actuel: les stratégies familiales dans le changement lié à l'immigration. Celles ci vont être interactives avec les niveaux vus précédemment et vont avoir pour fonction de permettre au groupe familial de vivre au mieux le changement. Articulant stratégies identitaires individuelles et modalités de négociation du changement dans la famille, elles seront le moteur du système familial dans ses rapports avec ses environnements réels ou idéels. Chaque membre de la famille portera ainsi sa part, jouera son rôle dans cette stratégie de groupe. Les indicateurs pertinents de ces stratégies familiales seront alors des comportements, attitudes, représentations véhiculés par chacun dans le sens où ils s'articulent à un fonctionnement, une règle du jeu familial (Minuchin: 1984).

Plus précisément, il nous paraît pertinent d'étudier de manière rigoureuse, pour chacun, en rapport avec le système familial, les aspects suivants:

- L'occupation du temps et sa représentation. Comment parents et enfants planifient-ils le temps social, le temps familial, le temps individuel? Quels en sont les rites collectifs et familiaux?

- La mobilisation des espaces (espaces individuels, sociaux, collectifs, familiaux) et leur internalisation. Comment la mobilité familiale dans l'immigration et le nouvel espace de la société d'accueil influencent-ils les modalités individuelles et familiales d'occupation de l'espacemaison, l'espace-ville, l'espace-école? Les notions d'intimité, de privé seront alors essentielles pour comprendre les représentations familiales.

- La topographie des réseaux primaires et secondaires d'affiliation et d'appartenance et la valeur qui leur est donnée. Comment la famille se situe-t-elle dans ses différents réseaux ? les réseaux du pays d’origine 
sont-ils survalorisés, dévalorisés? Quel est l'impact de cette représentation sur les réseaux actuels? isolement de la famille ou de certains de ses membres, suroccupation des réseaux de proximité, rapports défensifs vis-à-vis du réseau institué?

- Les modalités éducatives, leur transmission et leur réception. Comment articulent-elles les contraintes du pays d'accueil et les valeurs de chacun quant à l'enfance, à l'autorité, à la permissivité?

- L'organisation des rôles, statuts, fonctions dans la famille. Comment chacun participe-t-il à la reconstruction d'un équilibre familial selon les attentes et les contraintes à la fois de la culture d'origine et du pays d'accueil? Dans cette catégorie, les notions d'autonomie, de responsabilité, de devoir et de droit seront essentielles.

- Les processus de négociation familiale: comment se règlent les conflits, se prennent les décisions, se déroulent les communications.

Notre recherche exploratoire en cours au Québec nous permet déjà de dresser les grandes lignes de ces stratégies familiales, assez proches de celles perçues en France. Nous identifierons, dans chaque type de stratégies, différentes modalités de changement individuel et familial; cependant, toutes vont, selon notre première analyse, être reliées à un mode prévalent d'intégration au pays d'accueil.

Nous pouvons ainsi distinguer les stratégies offensives de promotion, qui s'effectuent à travers trois grandes modalités :

- L'entrée dans la compétition (travail - école) et l'assimilation opératoire de certaines valeurs (par exemple, l'individualisme pour réussir, l'autonomie pour être efficace). Les valeurs collectives traditionnelles continuent à être prônées et appliquées quand elles ne gênent pas le fonctionnement de la société d'accueil (hospitalité...). C'est alors principalement une promotion économique et sociale qui est recherchée par les différents membres de la famille.

- La participation communautaire (groupes et réseaux de la société d'accueil) et assimilation des valeurs par bénéfice individuel. Il s'agit là de promouvoir sa famille en lui donnant un statut reconnu par la société $d^{\prime}$ accueil. Les valeurs "autonomie, négociation formelle, droits et responsabilités » sont réajustées, intégrées au code traditionnel et deviennent moyen de valorisation sociale.

- La revendication d'identité ethnique (communauté ethnique et groupes de la société d'origine) et différenciation. Cette fois, c'est la différence qui est à promouvoir : il faut faire valoir sa communauté, son pays d'origine, la couleur de sa peau, comme un «plus » à respecter dans le pays d'accueil. 
Le second type de stratégies serait à visée défensive : nous les nommerons stratégies de repli, que celui-ci ait lieu sur la communauté ethnique, sur la famille, sur le pays d'origine idéalisé ou sur la religion. Ces stratégies se manifestent par une forme $d^{\prime}$ isolement familial ou groupal (ghetto), mais aussi par une peur de la société d'accueil vécue toujours comme menaçante. Les rapports aux institutions de cette société sont alors difficiles, éloignés, défensifs; ils se liront dans des comportements mésadaptés à l'école, au marché du travail, dans une distance linguistique soigneusement conservée, dans une formalisation extrême des rites traditionnels et dans la négation de certaines contraintes du pays d'accueil.

Enfin, les stratégies d'assimilation déculturante regrouperaient les fonctionnements familiaux visant à nier les différences vécues comme dévalorisantes et entraînant le conformisme des comportements et l'individualisme. Les effets à moyen terme de ces stratégies sont déstructurants pour le groupe familial, entraînant des ruptures dans les trajectoires individuelles et dans l'identité psychosociale des parents comme des enfants. Ces stratégies sont souvent liées à un vécu douloureux au moment de la décision d'immigration (réfugiés contraints au départ par la violence) ou avant (femmes avec leurs enfants rejetées par leur famille lors d'un divorce ou d'une répudiation). Elles constituent alors une suite à un processus de morcellement déjà en œuvre au pays. Elles peuvent aussi être dues à une vision excessive de l'intégration dans le pays d'accueil ( «il faut être comme eux, leur ressembler en tout »), l'adaptation entraînant alors une forme de reniement autocontraint du passé.

Si la notion de protection identitaire individuelle et collective est toujours centrale dans ces stratégies et si la famille continue à représenter I'organe protecteur par excellence de ses membres, les visées défensives et offensives nous paraissent représenter une vision bien particulière de l'intégration: dans le premier cas, il faut «faire sa place», dans le second, on ne peut que « défendre ses positions ». Ces dernières étant peu assurées dans la société d'accueil, c'est le plus souvent un espace marginal ou espace d'exclusion qui va être occupé comme base de repli.

Cette première grande classification des stratégies nous permet de pointer un concept sans doute central dans l'analyse à venir: celui de l'Estime de soi familiale en lien avec une Identité familiale en reconstruction du fait de l'immigration et de l'acculturation, qu'elle soit contrainte ou choisie.

La famille comme entité, comme institution et comme réseau de solidarité va être remise en question par l'immigration. La nouvelle identité familiale, dans une dynamique complexe et mouvante, va réarticuler les changements vécus, subis ou souhaités par chacun de ses membres.

Cette approche conceptuelle nous amène dès lors à réenvisager l'intervention sociale en milieu interculturel. 


\section{L'INTERVENTION SOCIALE FAMILIALE EN CONTEXTE INTERCULTUREL}

À travers ses stratégies d'acculturation, la famille va gérer au moindre coût énergétique et affectif l'articulation de quatre sous-systèmes culturels :

- celui du pays d'origine tel que connu avant le départ,

- celui du pays d'origine tel qu'il figure dans les représentations familiales après l'immigration,

- celui de la société d'accueil transmise par la communauté ethnique et son organisation,

- celui, enfin, de cette même société à travers ses institutions.

C'est dans cette gestion que l'intervenant social va intervenir, lui-même porteur des valeurs et modèles de la société d'accueil ou de la communauté ethnique dont il peut être membre. Et c'est à l'interaction, à la confluence de ces quatre sous-systèmes que l'intervention familiale prend tout son sens en contexte interculturel : il va en effet falloir travailler avec l'ensemble familial pour qu'il puisse maîtriser au mieux cette gestion et que celle-ci soit opératoire dans la société d'accueil. Mais il va falloir aussi permettre à chacun de comprendre son rôle dans ces stratégies, afin qu'un espace de possible s'ouvre dans cette complexité plus souvent vécue comme contraignante que comme innovante. La mission de l'intervenant social consistera en un accompagnement conscientisant du groupe familial et de chacun de ses membres dans ce parcours d'acculturation-changement.

L'accompagnement pourra alors être défini comme un cheminement de l'intervenant avec la famille et ses membres, à leur rythme, avec leurs modalités d'acculturation et leurs objectifs (Bouquet, 1992).

Les aidant à protéger leurs fondements identitaires individuels et collectifs, le travailleur social devra, dans cet accompagnement vers le changement, favoriser l'émergence de tactiques efficaces pour que chacun puisse à la fois se reconnaître et être reconnu par les autres dans ses multiples environnements, la famille étant le premier de ceux ci et pouvant agir comme catalyseur vers les autres.

Pour ce travail de conscientisation, l'intervenant utilisera des outils qui permettent d'analyser le quotidien : cartes de l'espace parcouru et occupé, schémas de trajectoires dans le temps, cartes des réseaux, génogrammes, écriture du roman familial, écriture du projet familial et des projets individuels. Ces différentes techniques pourront, selon la phase de l'intervention, être travaillées en individuel, dans le groupe familial ou dans des collectifs de familles.

En ce sens, si la croissance de l'estime de soi est souvent un objectif à atteindre en intervention individuelle, I'Estime de soi familiale pourra être un outil dans le choc culturel et dans la démarche de changement apportée par 
I'immigration. De même, L'Identité familiale, quand elle aura été définie et comprise dans ses rapports aux quatre sous-systèmes qu'elle articule, pourra être utilisée par chaque membre de la famille comme un support, un cadre ou un moteur selon ses propres stratégies de changement.

Dans ce contexte d'analyse et d'intervention, les ruptures, les éclatements, les pertes ne pourront sans doute pas être évités, mais ils seront accompagnés et compris non comme une simple contradiction entre la tradition et le modernisme, mais comme l'articulation complexe et spécifique que chacun fait entre hier et demain, entre soi et les autres, entre les différences et les convergences.

En guise de conclusion, il me paraît essentiel d'indiquer que ce modèle $d^{\prime}$ intervention, en construction, est issu des approches structurelle et écosystémique et qu'il en représente une application possible dans un contexte de changement et dans le champ de la famille. Il a déjà été pratiqué en France de manière informelle. Il est clair que la recherche en cours sur les stratégies familiales permettra de rendre ce type d'intervention à la fois plus efficace et plus systématique. La prise en compte de variables telles quela culture d'origine, les niveaux socioculturel et socioprofessionnel des parents, le lieu d'habitation (Montréal/régions éloignées), la présence et l'organisation des communautés ethniques s'avère dès maintenant nécessaire pour une analyse factorielle, à la fois qualitative et quantitative de ces stratégies et de leurs modalités.

\section{Références bibliographiques}

ABOU, A. S. (1981), L'identité culturelle. Paris: Antropos.

BOUCHARD, C. (1991), Un Québec fou de ses enfants. Rapport, Québec.

BOUQUET, B. (1992), «L'accompagnement professionnel », Revue PROJET.

CAMILLERI, C. (1980), "Les immigrés maghrébins de la seconde génération », Bulletin de psychologie, Paris, tome XXXIII, $\mathrm{n}^{\circ} 387$.

CAMILLERI, C. (1991), Stratégies d'identification. Paris: PUF.

CAMILLERI, C. (1992), «Inventaire de la situation des familles immigrées portugaises et maghrébines en Europe », Revue européenne des migrations internationales, Paris, vol. 8, $\mathrm{n}^{\circ} 2$.

CLAES, M. (1991), «Socialisation des adolescents en contexte multiethnique», dans Pluriethnicité, éducation et société : construire un espace en commun. Montréal : IQRC.

COSTA LASCOUX, J. (1986), "À propos de la délinquance des immigrés», dans Immigrations, multiethnicité et socialisation des jeunes. Paris: CRIV.

Duvignaud, J. (1979), L'anomie: hérésie ou subversion. Paris: Éditions du Seuil.

FORTIN, A. (1987), Histoires de familles et de réseaux. Québec: Éditions St-Martin. 
JACOB, A. et J. BERTOT (1991), Intervenir avec les migrants. Québec: Montréal : Éditions du Méridien.

JASMIN, C. (1992), Plus qu'une loi. Rapport, Québec.

LAPERRIÈRE, A. (1992), "Relations ethniques et besoins identitaires en contexte pluriculturel », dans Santé mentale au Québec. Montréal.

MALEWSKA PEYRE, H. (1982), Crise d'identité et déviance chez les jeunes immigrés. Vaucresson: CFRES.

MINUCHIN, S. (1984), Familles en thérapie. Paris : Éd. du Seuil.

VATZ-LAAROUSSI, M. (1986), "Transmission et changement culturels à travers les générations », dans Immigrations, multiethnicité et socialisation des jeunes. Paris: CRIV.

VATZ-LAAROUSSI, M. (1988), Les chômeurs de longue durée: acteurs ou exclus? Rapport de maîtrise, Université de Tours.

VATZ-LAAROUSSI, M. (1990), Étude des contrats d'insertion sur six départements de la région centre. Tours: LERFAS.

ZALESKA, M. (1982), "Identité culturelle des adolescents issus de familles de travailleurs immigrés», dans Crise d'identité et déviance chez les jeunes immigrés. Vaucresson: CFRES.

ZAvalloni, M. (1989), L'égo-écologie. Montréal : Éditions du Méridien. 